\title{
Mechanism of Relaxed Substrate Specificity in the Glycoside Hydrolase Family 5 Subclade 4
}

\author{
Christopher M. Bianchetti ${ }^{1}$, Jessica Anderson ${ }^{1}$, Samantha Kent $^{1}$, and Anthony Greco ${ }^{1}$ \\ ${ }^{1}$ Department of Chemistry, University of Wisconsin-Oshkosh, 800 Algoma Blvd, Oshkosh, \\ Wisconsin, 54901 \\ *Correspondence email: bianchec@uwosh.edu
}

Cellulosic biomass is an abundant and renewable resource that has the potential to become an inexpensive source of clean-burning fuels. However, the complex matrix of cellulose and hemicellulose found in plant cell walls are resistant to enzymatic and chemical deconstruction. Current commercial biomass degrading enzyme cocktails are composed of a complex mixture of glycoside hydrolases $(\mathrm{GH})$ that are difficult and costly to produce. One potential way to reduce the complexity of these cocktails is the incorporation of enzymes with relaxed substrate specificity, which we have termed multifunctional enzymes. Multifunctional GHs hydrolyze the glycosidic bonds found in the three major components of plant cell walls (cellulose, mannan and xylan) with similar specific activity. Previous work has shown that the GH family 5 subclade 4 (GH5.4) is enriched in multifunctional GHs. To elucidate the structural features that cause multifunctionality the crystal structures of five multifunctional and four nonmultifunctional GH5.4 enzymes were determined. Structural alignments of the high-resolution GH5.4 structures showed that the substrate binding clefts are highly similar, suggesting that small structural perturbations are responsible for relaxed substrate specificity. Eight structurebased variants of the multifunctional GH5.4 enzyme from Ruminococcus flavefaciens FD-1, designated here as RfCMX, were generated and the enzymatic activity of these mutants are under investigation. Additionally, the binding affinity of RfCMX for cellohexaose, mannohexaose and xylohexaose was determined using isothermal calorimetry. RfCMX binds cellohexaose and xylohexaose with similar affinity. However, RfCMX binds cellohexaose approximately 100 fold tighter than mannohexaose. Identification of the amino acid residues responsible for multifunctionality would further the understanding of how GHs interact with cellulosic biomass and elucidate the mechanism of multifunctionality observed in GH5.4 enzymes. 